\title{
Stress partitioning in a near- $\beta$ Titanium alloy induced by elastic and plastic phase anisotropies: experimental and modeling
}

\author{
Ravi raj purohit PURUSHOTTAM RAJ PUROHIT ${ }^{1,2 *}$, Safaa LHADI ${ }^{1,4}$, Nathalie GEY ${ }^{1,4}$, Olivier CASTELNAU ${ }^{3}$, Thiebaud \\ RICHETON $^{1,4}$, Lionel GERMAIN ${ }^{1,4}$, Stephane BERBENNI ${ }^{1,4}$ \\ *ravi.purushottam-raj@irt-m2p.fr
}

${ }^{1}$ Laboratoire d'Étude des Microstructures et de Mécanique des Matériaux, Université de Lorraine, CNRS, Arts et Métiers Paris Tech, LEM3, F-57000 Metz, France

${ }^{2}$ Institut de Recherche Technologique Materiaux, Metallurgie, Procedes (IRT M2P), 4 Rue Augustin Fresnel, 57070 Metz, France

3 PIMM, CNRS UMR 8006, Arts et Métiers ParisTech, CNAM, 151 Bd de l'Hôpital, 75013 Paris, France

${ }^{4}$ Laboratory of Excellence on Design of Alloy Metals for Low-mAss Structures (DAMAS), Université de Lorraine, France

\begin{abstract}
The load transfer induced by the elastic and plastic phase anisotropies of a Ti-10V-2Fe-3Al titanium alloy is studied. The microstructure consists in a nodules embedded in elongated $\beta$ grains. EBSD performed on the alloy shows no crystallographic texture neither for $a$ nor $\beta$ phase. Tensile tests along the elongation direction, at a strain rate of $2 \times 10^{-3} \mathrm{~s}^{-1}$ give a yield stress of 830 MPa with $13 \%$ ductility.

Simulations based on an advanced two-phase polycrystalline elasto-viscoplastic self-consistent (EVPSC) model predict that the $\beta$ phase first plastifies with a sequential onset of plasticity starting from $<110>$ oriented $\beta$ grains, then $<111>$ and finally $<100>$ oriented $\beta$ grains. This leads to a strong load transfer from the $\beta$ grains to the a nodules whose average behavior remains elastic up to high stresses ( 940 MPa). However, additional simulations considering exclusively $\beta$ grains of specific orientation show that the behavior of a nodules is strongly dependent on the $\beta$ texture in which they are embedded. Especially, in $<001>\beta$ grains, which plastify the latest, the model predicts the onset of plasticity in favorably orientated a nodules. Moreover, the orientation spread within the $\beta$ grains can modify the average plastic behavior of a phase. In future, these results will be compared to data obtained from in-situ High Energy XRD and SEM/EBSD experiments.
\end{abstract}

\section{Introduction}

Near $\beta$ alloys like Ti-10-2-3 are often used for aircraft structural applications [1-3] due to their excellent forgeability and mechanical properties at low temperatures. These alloys undergo a complex transformation process, including $\beta$ and $\alpha / \beta$ forging. The final microstructure differs based on the thermomechanical history and the fraction of alloying elements [4]. Generally, it consists of a nodules embedded in a $\beta$ matrix partially transformed during aging to secondary $a_{\mathrm{s}}$ platelets of nanometric size [1].

The elastic-plastic behavior of these near $\beta$ alloys is rather complex. Even the anisotropic elastic behavior is not fully understood. Literature shows a wide spread for the anisotropic elastic constants of $\beta$ phase with Zener anisotropy coefficient ranging from 1.4 to 8.3 [5]. This can possibly be due to chemical compositions of the corresponding phase evolving with the thermal history, although one cannot sweep out the difficulties to measure local elastic constants in a multiphase polycrystalline specimen. Such lack of accuracy impedes predictive understanding of the material behavior. Grain-scale elastic and plastic anisotropies of both $\beta /$ a phases often lead to stress partitioning and to heterogeneous onset of plasticity [6]. They must be understood as they strongly influence the macroscopic elasto-viscoplastic behavior and the associated development of internal stresses that are responsible 
for isotropic and kinematic hardening as well as crack initiation. For these alloys, significant cracks have been observed after failure originating from basal slip activity in the a nodules $[7,8]$.

Mean field Self-Consistent models have been successfully used in the past together with experimental techniques like X-ray diffraction (XRD) to characterize the stresses and strains in complex materials by taking into account grain and phase interactions [9]. Many studies have been carried out in combination with in-situ diffraction experiments and self-consistent schemes to characterize the plastic deformation in titanium alloys [10 - 13]. These studies employ elasto-plastic or viscoplastic models and predict the average behavior of the phases reasonably well.

The present work aims to predict the inter-granular stress and strain evolution in the forged Ti-10-2-3 alloy during both elastic and plastic deformations. For this purpose, an advanced two-phase polycrystalline elasto-viscoplastic self-consistent model, with first order "affine" linearization of the viscoplastic flow rule, as described in [5] is applied on a simplified microstructure, i.e. a nodules embedded in a $\beta$ matrix, as the first step. Alongside, an attempt is made to illustrate the role of anisotropic elastic $\alpha / \beta$ strains on the hierarchical onset of plasticity. The simulated data will be compared in the future to high energy XRD results.

\section{Experimental procedure}

\section{II.1 Material:}

Samples were cut from an $\alpha / \beta$ forged piece of the 10-2-3-titanium alloy. They were all heat treated in the $\alpha / \beta$ domain for 1 hour and water quenched to obtain a nodular microstructure with $15 \%$ of primary a nodules embedded in a $\beta$ matrix. This matrix consists of millimeter large elongated $\beta$ grains, which are partially fragmented into equiaxed sub-grains with average size of 2.7 $\mu \mathrm{m}$. The primary a nodules are present both at the $\beta$ grain and sub-grain boundaries. Their average size is around $1.2 \mu \mathrm{m}$. Further observation of the microstructure also confirmed that no secondary a lamellae formed during water quenching. The a volume fraction was confirmed to be around $\sim 15 \%$ using laboratory $X$-ray diffraction.

\section{II.2 Texture and Microtexture:}

Neutron texture analysis was carried out on $1 \mathrm{~mm}$ cube samples at Laboratoire Léon Brillouin (LLB) in France. Pole figures were generated for the $\beta$ phase and the crystallographic texture was found to be random (not shown here). Additional EBSD measurements were performed on a JEOL JSM-6500F electron microscope equipped with the Nordlys-S camera. Several EBSD maps with $0.5 \mu \mathrm{m}$ step size were carried out along the cross-section (normal to the elongation axis) to characterize the microstructure and local texture of $a$ and $\beta$ phases. The EBSD maps were post-processed with Aztec-Oxford instrumentation software.

Figure 1a shows the $\beta$ - inverse pole figure (IPF) map of along the elongation direction. Figures $1 \mathrm{~b}$ and $\mathrm{c}$ give a close-up view of the rectangular area marked in Figure 1a to highlight the misorientations among $\beta$ sub-grains (Figure 1b) and the phase map showing the primary $a$ nodules in red and $\beta$ grains in blue (Figure 1c). Equiaxed $\beta$ sub-grains were found throughout the microstructure with both small $\left(>3^{\circ}\right)$ and large $\left(>15^{\circ}\right)$ misorientations. A higher fraction of high angle grain boundaries is observed within a single $\beta$ grain and this forms the basis of equiaxed morphological assumption for the $\beta$ grains. Along with the high angle grain boundary sub-grains, the $\beta$ grains also present a significant orientation spread. The a nodules in the $\beta$ grains are mostly equiaxed and their texture is very close to random, i.e. the orientation relationship with the $\beta$ phase was broken.

\section{II.3 Tensile test:}

Cylindrical tensile specimens with $6.35 \mathrm{~mm}$ diameter were cut from the $\alpha / \beta$ forged piece along the elongation direction (the tensile direction TD is parallel to the elongation direction of the $\beta$ grains). The width of the $\beta$ grains is $\sim 200 \mu \mathrm{m}$, such that there is $\sim 100$ grains in the tensile specimen's cross-section. The tensile tests were performed on a ZWICK Z250 machine with a force cell of $250 \mathrm{kN}$ and a contact extensometer. Several different strain rates were studied $\left(2 \times 10^{-5} \mathrm{~s}^{-1}, 2 \times 10^{-4} \mathrm{~s}^{-1}, 2 \times 10^{-3} \mathrm{~s}^{-1}\right)$ up to fracture. The results are more detailed in [14]. In this work, only the tensile response at $2 \times 10^{-3} \mathrm{~s}^{-1}$ is shown and used for the present micromechanical simulations. 


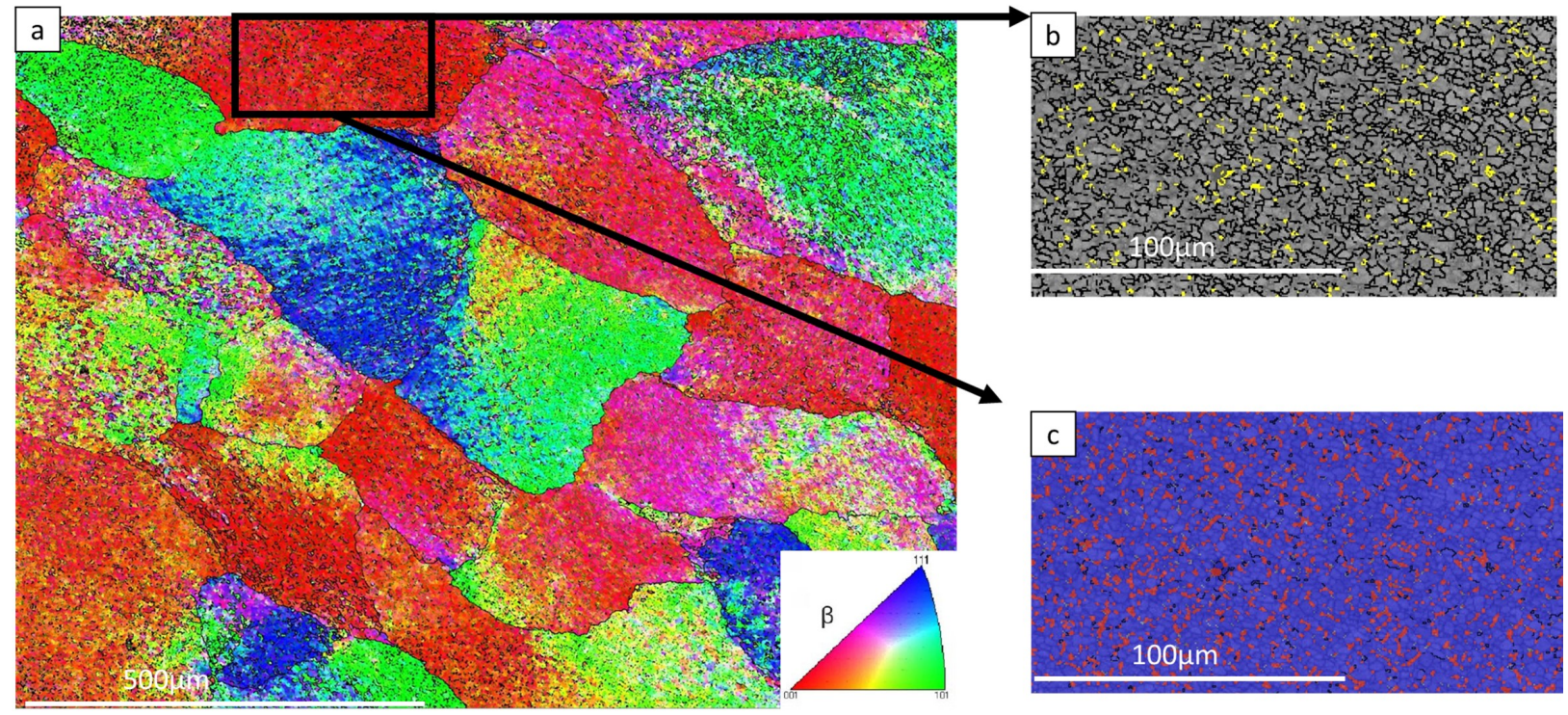

Figure 1 (a) $\beta$-IPF map along the elongation direction (normal to the acquisition surface). (b) $\beta$ - misorientation map of a region inside a $\beta$ grain (black and yellow lines indicate grain boundaries for respective misorientations $>3^{\circ}$ and $>15^{\circ}$ ). (c) phase map ( $\alpha$ and $\beta$ phases are shown in red and blue colors).

\section{Affine-based Elasto-Visco-Plastic-Self-Consistent modeling}

\section{III.1 Single grain constitutive behavior in $\alpha / \beta$ phases:}

A one-site elasto-viscoplastic self-consistent (EVPSC) scheme, formulated using the translated field method and a first order affine approximation, is used. It has been described in detail elsewhere [5, 15] and only a brief description of the single crystal law employed is presented. The simulations are performed under the infinitesimal small strain assumptions, where the total strain rate tensor $\left(\dot{\epsilon}^{e}\right)$, calculated from the generalized Hooke's law, and the nonlinear viscoplastic strain rate tensor $\left(\dot{\epsilon}^{v p}\right)$.

$$
\dot{\epsilon}=\dot{\epsilon}^{e}+\dot{\epsilon}^{v p}
$$

The constitutive behavior at the single crystal level, for both $\alpha$ and $\beta$ phases, is described by Méric-Cailletaud (MC) model [16]. The slip rate $\dot{\gamma}^{s}$ for each slip system 's' is defined as:

$$
\dot{\gamma}^{s}=\left\langle\frac{\left|\tau^{s}-x^{s}\right|-r^{s}}{K}\right\rangle^{n} \operatorname{sign}\left(\tau^{s}-x^{s}\right)
$$

Where $=\max (\mathrm{x}, 0), \tau^{\mathrm{S}}$ is the resolved shear stress defined as $\tau^{\mathrm{S}}=\mathrm{R}^{\mathrm{S}}: \sigma$, with $\mathrm{R}^{\mathrm{S}}$ is the symmetric Schmid orientation tensor and $\sigma$ is the first moment of stresses (average stress inside a given grain, i.e. a given orientation). The quantities $x^{s}$ and $r^{s}$ are the kinematic and isotropic hardening components on each slip system, respectively. The evolution of the kinematic hardening is described through a simplified law with a single material coefficient c:

$$
\dot{x}^{s}=c \dot{\gamma}^{s}
$$

The viscoplastic strain rate at the scale of grain is defined as:

$$
\dot{\epsilon}^{v p}=\sum_{s=1}^{N} R^{s} \dot{\gamma}^{s}
$$

III.2 EVPSC scale transition law:

The EVPSC scale transition law from micro- to macroscopic fields as detailed in [5] was used in this paper. Other details and comparisons with other mean/full field approaches can be found in [17].

\section{III.3 Application of the affine EVPSC to Ti-10-2-3:}

Two types of simulations have been carried out: the first one at the macroscopic scale and the second one at the scale of individual $\beta$ grains. These simulations are carried out assuming equiaxed grain morphology for both $\alpha$ and $\beta$ phases with volume 
fraction coming from the XRD analysis ( $15 \%$ of a phase).

Simulation I: An isotropic crystallographic texture is considered for both the $\beta$ and a phases, as observed experimentally with neutron texture analysis. Several simulations were performed to identify the number of orientations required in each phase to obtain an isotropic elastic behavior (as observed experimentally from tensile tests in different directions - not shown here) [14]. It was found that at least 5000 random orientations are required in the a phase and 2000 random orientations are required for the $\beta$ phase. The Representative Volume Element (RVE) is a polycrystalline $(\alpha+\beta)$ composite with 7000 random orientations.

Simulation II: The second simulation is performed at the scale of single $\beta$ grains with $\alpha / \beta$ texture coming from the EBSD map. The choice of specific $\beta$ grains oriented close to the $<100>$ || TD was motivated from the results of Simulation I. Indeed, those grains were seen to be the latest to enter to plasticity with the model. They are hereafter referred as 'Red grains' (with respect to the Standard Stereographic Triangle (SST) color code). Figure 2 shows the choice of six 'Red grains' on the EBSD map (selection of grains based on high-angle boundary with misorientation angle of $>25^{\circ}$.). The six red grains present local spread in orientation; for example, red grain 2, 5 and 6 exhibit lower spread in orientation locally, while the red grain 1, 3 and 4 presents significant spread in orientation. As mentioned previously, the texture of the a phase in these 'Red grains' is rather random.

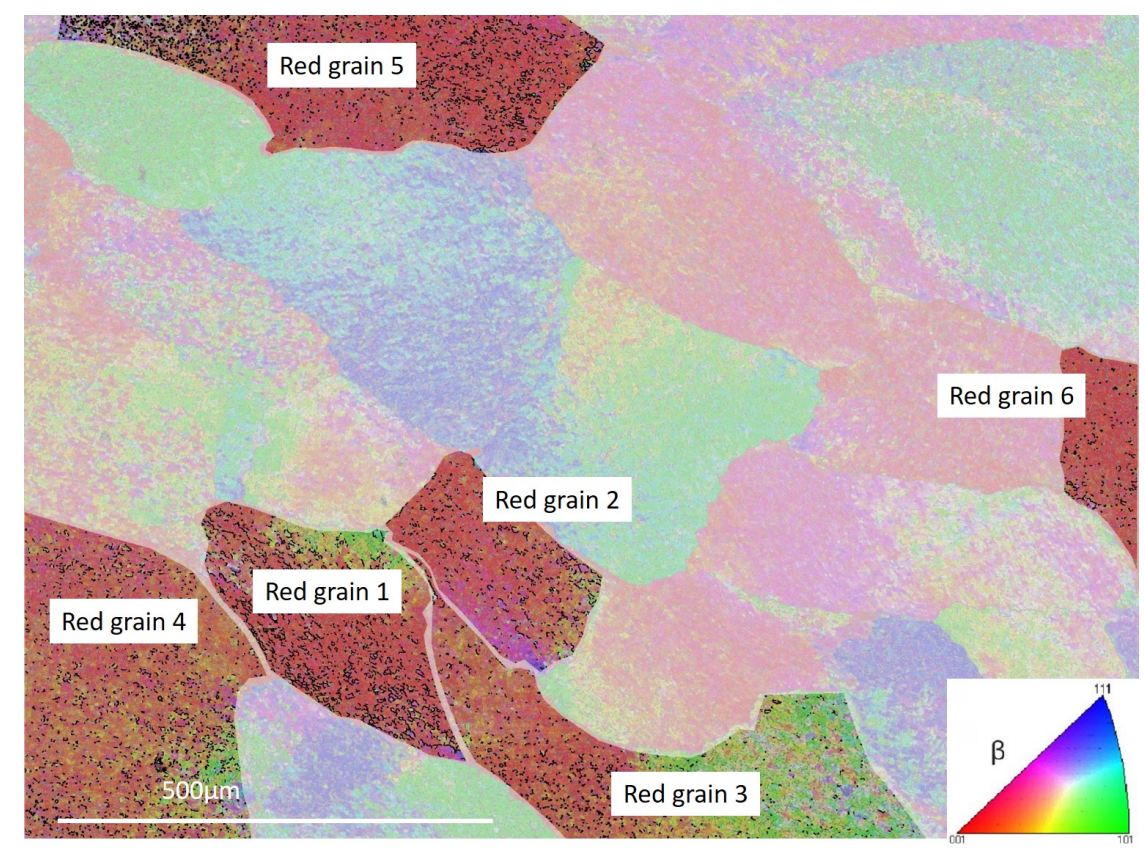

Figure 2 Selection of six 'red grains' on the $\beta$-IPF map along the elongation direction (normal to the acquisition surface).

\section{III.4 Elastic and viscoplastic parameters:}

The elastic and viscoplastic parameters have been fitted on experimental tensile curves at several strain rates. The parameters used for both simulations I and II are presented in Table 1.

The elastic constants for the a phase have been selected from the literature [18], as there is considerable agreement. However, for the $\beta$ phase, widely different elastic constants have been reported in the literature with an anisotropic factor ranging from 1.4 to 8.3. They have a significant influence on the predicted elastic and plastic incompatibilities at the grain scale as discussed in [5] for a 10-2-3-Ti alloy fully $\beta$. Here, we consider the same constants for $b$ phase as used by Martin et al. [19], as it provides reasonably good fit with the experimental data for a $100 \% \beta$ phase alloy. The parameter ' $n$ ' is chosen to be relatively high ( $n=90$ for the a phase and $n=80$ for the $b$ phase) to predict the observed material's low strain rate sensitivity (see [14]). For the a phase, the deformation proceeds by basal, prismatic and first order pyramidal slip, while for the $b$ phase the $\{110\}<111>,\{112\}<111>$ and $\{123\}<111>$ slip modes are used. The critical resolved shear stresses for these slip systems were estimated based on the good fit with the experimental macroscopic stress-strain curve and the observation of basal slip lines predominance in a nodules in 10-23-Ti alloys $[7,20]$. 
It should be noted that the investigated microstructure is a simplified one with primary a nodules embedded in a $\beta$ matrix (with no secondary a lamellae). The chosen SEC and viscoplastic parameters for the model are expected to change for the classical aged microstructure in which the $\beta$ matrix is partially transformed into fine secondary a lamellae. A recent experimental study [7] on the $\beta$ metastable Ti-5Al-5Mo-5V-3Cr has also highlighted the role of elastic anisotropy of the transformed $\beta$ phase on the elastic and plastic behavior.

\section{III.5 Data analysis and interpretation:}

Along with the conventional macroscopic curves, our simulations were also applied to predict the evolutions of the elastic strains with applied stress and their deviation from linearity to identify the onset of micro-plasticity and load transfers between $a$ and $\beta$ phases. The interpretation of elastic strain evolution is taken from the XRD lattice strain analysis [21]. By following the evolution of lattice (i.e. purely elastic) strains with applied stress in a composite material, one can identify the plastic events and load transfer amongst different phases/grain families. A deflection of elastic strains from linearity, when plotted with respect to applied stress, indicates the nature of deformation of the phase. A negative deflection from linearity indicates plasticity, whereas a positive deviation indicates elastic load takeover or "load-transfer" from the grain family/phase that has achieved plasticity.

Table 1 Elastic and viscoplastic parameters of the $\alpha$ and $\beta$-phases for each slip system family.

\begin{tabular}{|c|c|c|c|c|c|c|c|}
\hline Parameters & \multicolumn{4}{|c|}{$\boldsymbol{\alpha}(\mathrm{hcp})$} & \multicolumn{3}{|c|}{$\boldsymbol{\beta}(\mathrm{bcc})$} \\
\hline $\begin{array}{c}\text { Elastic moduli } \\
\text { (anisotropic) } \\
\text { (GPa) }\end{array}$ & \multicolumn{4}{|c|}{$C_{11}=160, C_{33}=181, C_{44}=46.5, C_{12}=90, C_{13}=66$} & \multicolumn{3}{|c|}{$\mathrm{C}_{11}=100, \mathrm{C}_{12}=70, \mathrm{C}_{44}=36$} \\
\hline \multirow{2}{*}{$\begin{array}{l}\text { Viscoplastic and } \\
\text { hardening } \\
\text { parameters }\end{array}$} & \multicolumn{4}{|c|}{ Slip system families } & \multicolumn{3}{|c|}{ Slip system families } \\
\hline & Basal & Prismatic & Pyramidal $<\mathrm{a}>$ & Pyramidal $<\mathrm{c}+\mathrm{a}>$ & $\begin{array}{l}\{110\} \\
<111>\end{array}$ & $\begin{array}{l}\{112\} \\
<111>\end{array}$ & $\begin{array}{l}\{123\} \\
<111>\end{array}$ \\
\hline $\mathbf{n}$ & 90 & 90 & 90 & 90 & 80 & 80 & 80 \\
\hline K $\left(\right.$ MPa.s $\left.^{1 / n}\right)$ & 270 & 270 & 270 & 270 & 300 & 3000 & 300 \\
\hline $\mathbf{r}^{\mathbf{s}}(\mathbf{M P a})$ & 215 & 350 & 435 & 620 & 70 & 70 & 80 \\
\hline $\mathbf{c}^{\mathrm{s}}(\mathrm{MPa})$ & 0 & 0 & 0 & 0 & 200 & 200 & 400 \\
\hline
\end{tabular}

\section{Results and Discussion:}

\section{IV.1 Simulation I:}

The simulation is carried out on the $\alpha+\beta$ composite with 7000 random crystal orientations, up to a total strain of $10 \%$ (below the failure strain of the material). The predicted $\alpha+\beta$ composite response along with the experimental curve for $2 \times 10^{-3} \mathrm{~s}^{-1}$ strain rate is shown in Figure 3. The elastic response is well reproduced but a small discrepancy could be seen regarding the elastic-plastic transition. The difference in yield stress predicted by the model is about $40 \mathrm{MPa}(\sim 5 \%$ higher than experimental data). However, these discrepancies were found to lie within the scatter of tensile data for different tested specimens of close microstructures. 


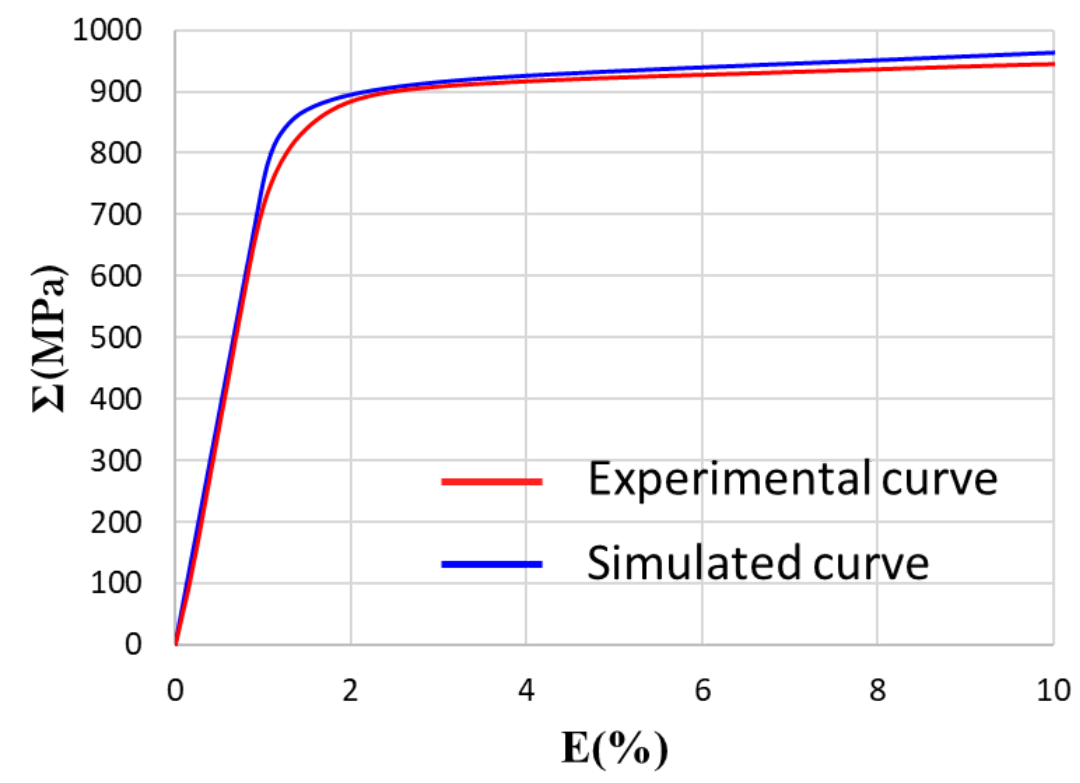

Figure 3 Experimental and predicted macroscopic stress-strain response of Ti-10-2-3 (random texture) at a strain rate of $2 \times 10^{-3} \mathrm{~s}^{-1}$.

The evolution of elastic strains with applied stress as well as its deviation from linearity is given in Figures $4 a$ and $4 b$, for the whole composite (in black) and for the $\beta$ and $a$ orientations separately (respectively in blue and red). Figure $4 b$ indicates the onset of plasticity in $\beta$ phase with a strong load transfer to the a nodules. The behavior of the vast majority of a nodules remains elastic (see Figure $4 \mathrm{~b}$ and section III.4 for interpretation of the curves). As will be shown in simulation II, the behavior of a nodules is however strongly dependent on the $\beta$ texture in which they are embedded.

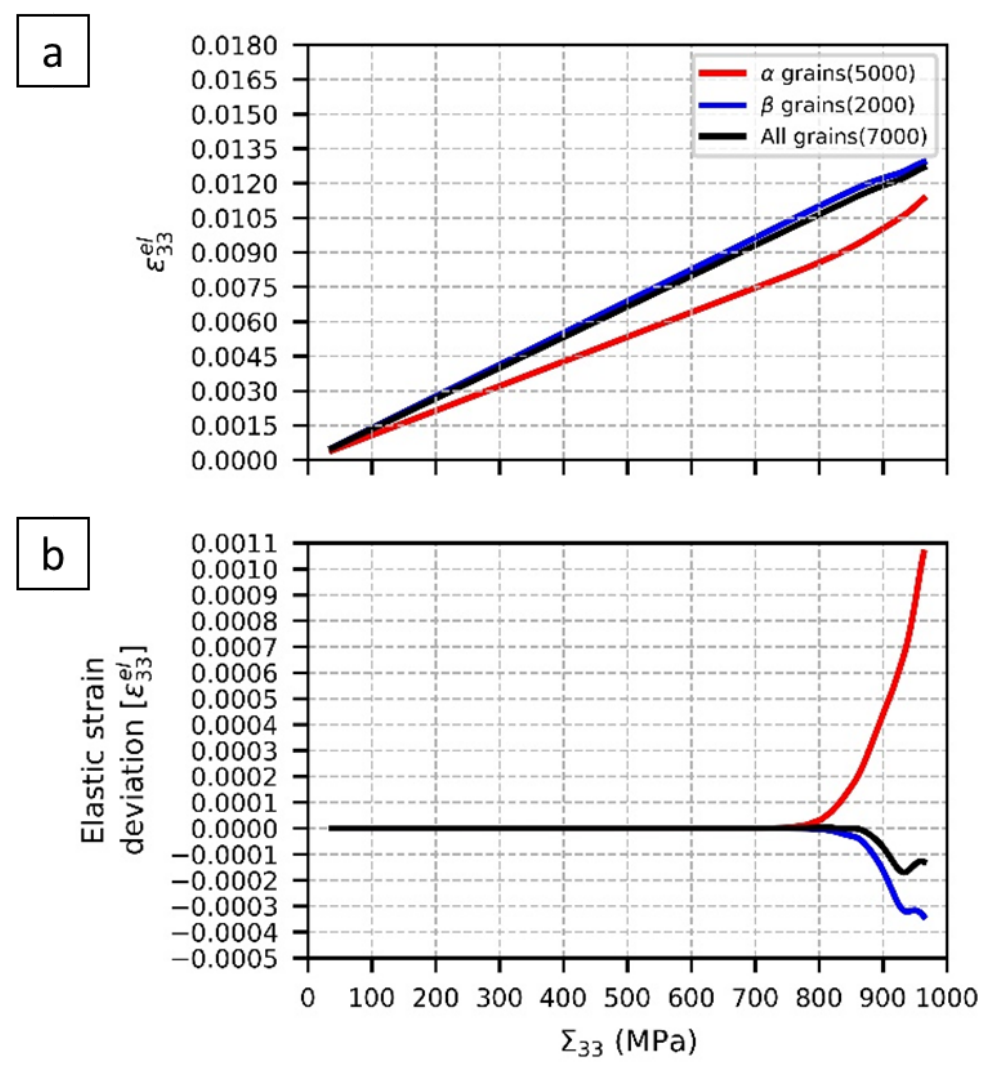

Figure 4 (a) Evolution of elastic strain with respect to applied stress for $\alpha+\beta$ composite. (b) Elastic strain deviation from linearity versus applied stress (linear fits are carried out for data points below elastic region: $400 \mathrm{MPa}$ ). The numbers in the closed brackets in the legend indicate the number of grains. 
The analysis by grouping different subsets of $\beta$ fibers along TD indicates a sequential onset of plasticity starting from <110> oriented $\beta$ grains with load transfer to $<100>$ grains, see Figure 5 . At higher stresses, plasticity is seen to set in for the $<111>\beta$ grains family subset and finally for the $<100>\beta$ grains. On the average elastic strain curve for $\beta$ phase (black solid line in Figure $5 c$ ), the macro-plasticity in $\beta$ phase starts when all the three-grain families' subset has attained plasticity (i.e. 860 MPa).

This sequential onset of plasticity in the $\beta$ phase predicted by our simulation agrees well with the in-situ XRD observations on the forged Ti-10-2-3 (similar microstructure) by Raghunathan and coworkers [10].
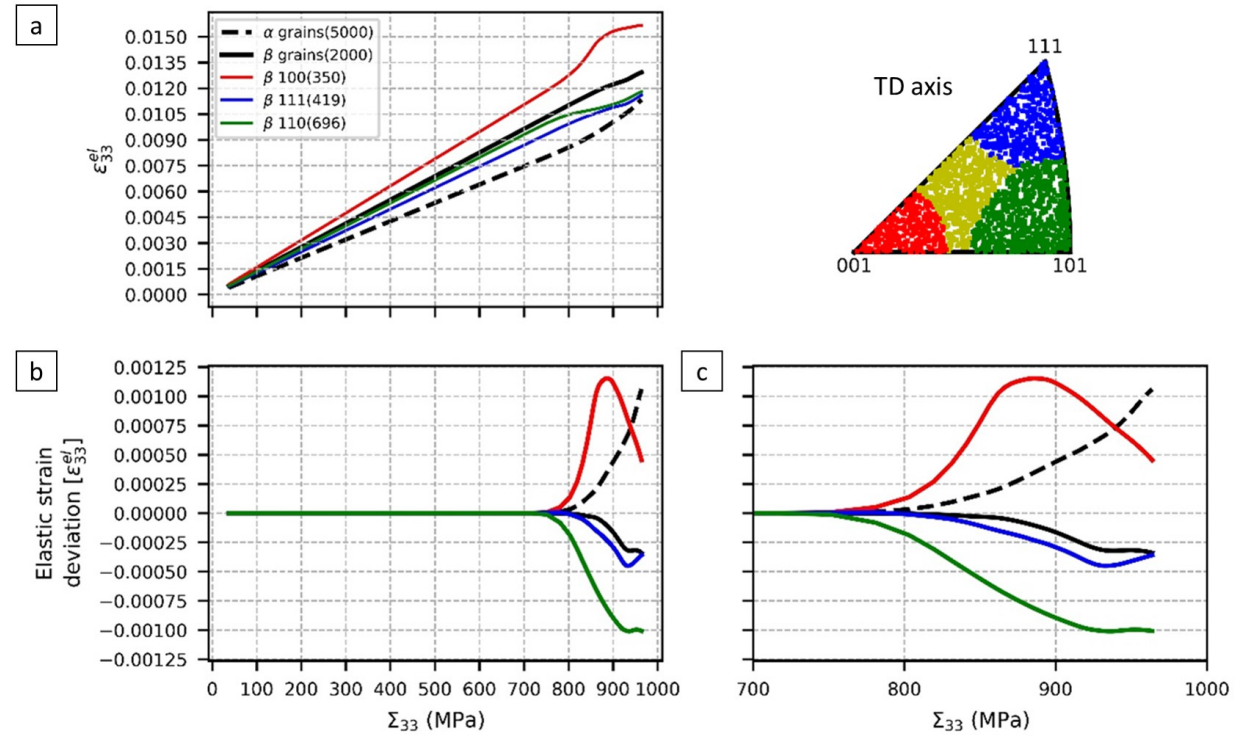

Figure 5 (a) Evolution of elastic strains with applied stress for $\alpha+\beta$ composite, with $\beta$ grain family subsets. (b) Elastic strain deviation from linearity versus applied stress (linear fits are carried out for data points below elastic region: $400 \mathrm{MPa}$ ). (c) Magnified section of the elastic strain deviation plot. The colors in the plot correspond to the color-coded SST provided alongside.

\section{IV.2 Simulation II:}

It was seen in Simulation I that for the $\beta$ phase, the $<100>$ || TD oriented grains enter to a plastic regime at the end. Hence, several $\beta<100>||$ TD oriented grains were selected from the EBSD map to predict the average behavior of the embedded a nodules (see Figure 2). For clarity, only the results of red grains 1, 3 and 6 will be discussed due to the significant presence of local orientation spread.

Contrary to Simulation I, an inverse trend is seen for the onset of plasticity and load transfer in Simulation II, see Figure 6b. The onset of plasticity is now seen in the a phase first, with a negative deviation of elastic strains from linearity, with load transfer to $\beta$ grains. At higher applied stresses (770-800 MPa), plasticity is attained in the $\beta$ phase, resulting in a load transfer with the a phase (Figure 6b). Further plasticity is seen in the a phase with second load transfer to $\beta$ phase for red grains 1 and 6 ( $810-830 \mathrm{MPa}$ ). However, in the case of red grain 3 , the second load transfer to the $\beta$ phase is not seen.

The plastic behavior of extremal Young's moduli oriented a nodules has also been analyzed (see Figure 6c). For the a nodules with ' $c$ ' axis parallel to tensile direction (unfavorable orientation -for both basal and prismatic systems), no plastic strain could be seen. However, for the ones having the ' $c$ ' axis perpendicular to the tensile direction (unfavorable orientation of basal systems, favorable orientation of prismatic systems), plasticity is seen in the red grains 1 and 6 . It should be noted that the onset of prismatic slip is seen only after plasticity has been set in the $\beta$ grain.

Thus, simulation II highlights two major results: (1) the plastic behavior of a nodules depends on the $\beta$-orientation it is embedded in and on the orientation of the nodule itself, (2) having a $\beta$ grain with a large orientation spread (red grain 3) can result in modified average plastic behavior of a phase compared to a $\beta$ grain with less orientation spread (red grain 1 and 6 ). 
a

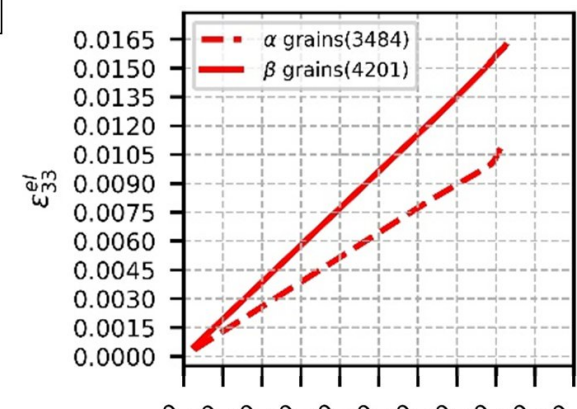

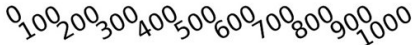

$\Sigma_{33}(\mathrm{MPa})$

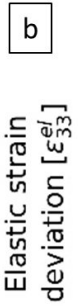

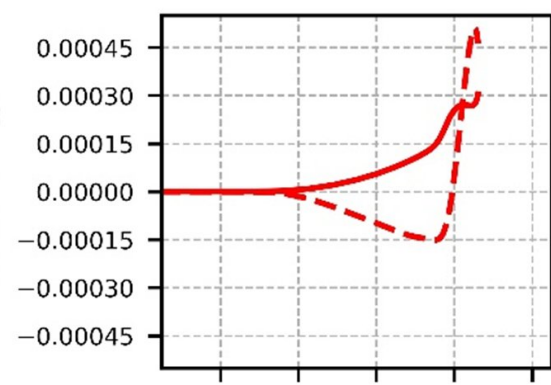

$5^{60} \quad 6^{40} \quad 120 \quad 8^{00} \quad 8^{80}$

C

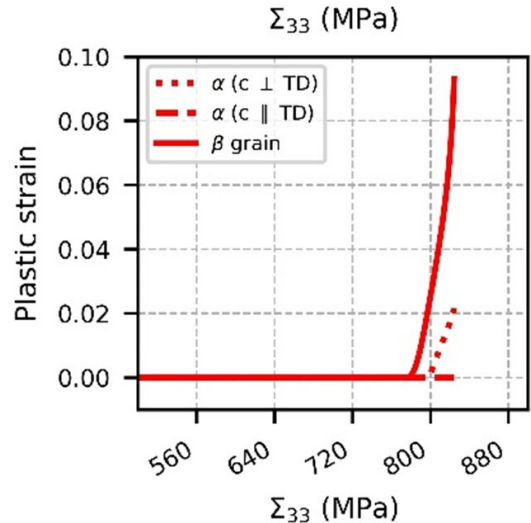

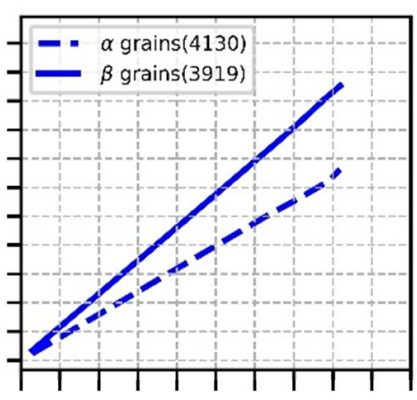

$\mathrm{O}_{1} \mathrm{OO}_{2} \mathrm{OO}_{3} \mathrm{OO}_{4} \mathrm{OO}_{5} \mathrm{OO}_{6} \mathrm{OO}_{7} \mathrm{OO}_{8} \mathrm{OO}_{9} \mathrm{O}_{1} \mathrm{O}_{\mathrm{O}}$

$\Sigma_{33}(\mathrm{MPa})$

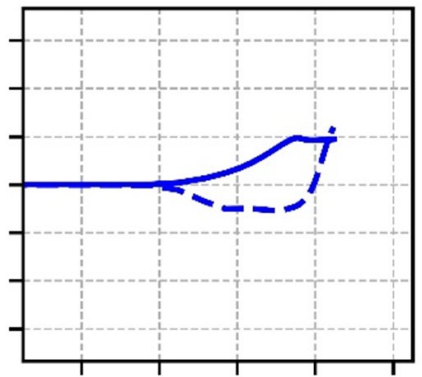

$5^{60} \quad 6^{40} \quad 120 \quad 800 \quad 88^{\circ}$

$\Sigma_{33}(\mathrm{MPa})$

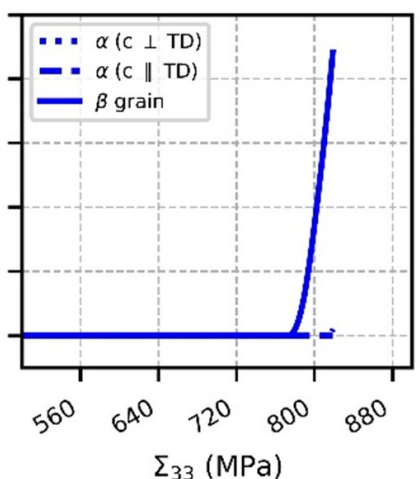

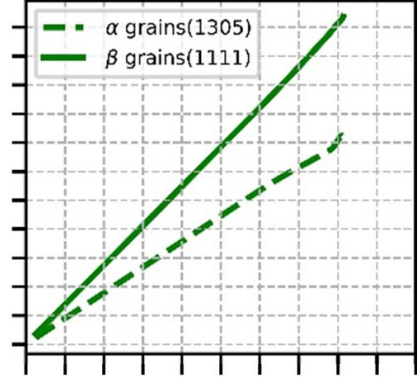

$\mathrm{O}_{2} \mathrm{OO}_{2} \mathrm{OO}_{3} \mathrm{OO}_{4} \mathrm{OO}_{5} \mathrm{OO}_{6} \mathrm{OO}_{7} \mathrm{OO}_{8} \mathrm{OO}_{3} \mathrm{O}_{2} \mathrm{OOO}^{\circ}$ $\Sigma_{33}(\mathrm{MPa})$

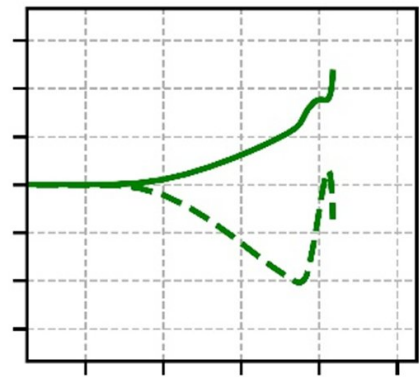

$5_{50}^{60} \quad 6^{40} \quad 120 \quad 80^{\circ} \quad 8^{80}$

$\Sigma_{33}(\mathrm{MPa})$

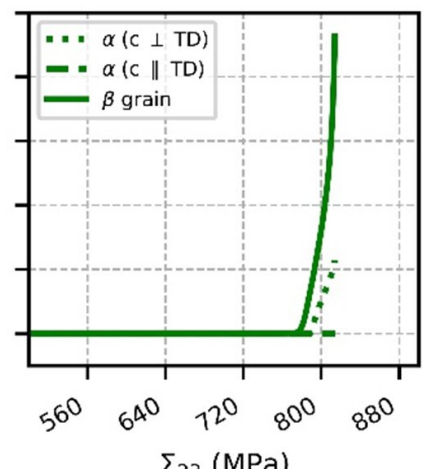

$\Sigma_{33}(\mathrm{MPa})$

Figure 6 (a) Evolution of elastic (lattice) strain with applied stress for $\alpha+\beta$ for Red grains 1, 3 and 6 . (b) Elastic strain deviation from linearity versus applied stress (linear fits are carried out for data points below the elastic region: $400 \mathrm{MPa}$ ). (c) Plastic strains with applied stress in the extremal Young's modulus orientation for $\alpha$ and $\beta$.

\section{Conclusions and perspectives}

An EVPSC model was applied to simulate the tensile behavior and the associated stress partitioning for a $(\alpha+\beta)$ polycrystalline Ti10-2-3 alloy with a forged microstructure (primary a nodules embedded in a $\beta$ matrix). The real texture and local texture of each phase was measured using neutron diffraction and EBSD acquisitions. The numerical results indicate that the onset of plasticity in a phase could be influenced by the crystallographic texture of the other phase.

- At the scale of the specimen: the $\beta$ phase is predicted to plastify first with sequential onset of plasticity starting from $<110>||$ TD, then <111> || TD and finally <100> || TD grains. This is followed by a strong load transfer to the a phase.

- At the scale of $\beta$ grains (<100> || TD oriented $\beta$ grains with rather random oriented a nodules): it is predicted that plasticity is initiated in the $\alpha$ phase first with a load transfer to the $\beta$ phase. The plastic behavior of $a$ phase was found to depend on the $\beta$ grain in which it is embedded and its orientation spread.

High energy X-ray diffraction experiments are planned by the end of this year to obtain lattice strains evolution with applied stress and to verify the sequential onset of plasticity and corresponding load transfer amongst phases. It is also expected to refine the anisotropic elastic constants for the $\beta$ phase and the viscoplastic/hardening parameters of the model from lattice strain evolution for different orientations in both phases. In-situ SEM studies are also planned to verify slip system activity (at least in the $a$ phase) to check if the active slips suggested by the EVPSC model are in good agreement with experiment. 


\section{Acknowledgments:}

The authors are thankful to Dr. Julien Capelle (LEM3) for his help with the mechanical tests. Computations were performed on supercomputer facilities hosted by Laboratoire d'étude des Microstructures et de Mécanique des Matériaux (LEM3). This work is supported by the French State through two programs operated by the National Research Agency (ANR) (1) "Investment in the future" referenced by ANR-11-LABX-0008-01 (Laboratory of Excellence 'DAMAS': Design of Alloy Metals for low-mAss Structures) and (2) "Plan d'Investissement d'Avenir" (PIA) in the frame of a research program managed by "Institut de Recherche Technologique Matériaux, Métallurgie, Procédés" (IRT M2P).

\section{References:}

[1] Chini, M R, et al. "Advanced Microtexture Analysis of a Ti 10-2-3 Product for Better Understanding of Local Variations in Mechanical Behavior." Proceedings of the 13th World Conference on Titanium. Hoboken, NJ, USA: John Wiley \& Sons, Inc., 2016.

[2] Uta, E., et al. "Texture heterogeneities in ap/as titanium forging analysed by EBSD-Relation to fatigue crack propagation." Journal of microscopy 233.3 (2009): 451-459.

[3] Gey, N., et al. "Texture and microtexture variations in a near-a titanium forged disk of bimodal microstructure." Acta Materialia 60.6-7 (2012): 2647-2655.

[4] Settefrati, A, et al. "Precipitation in a near beta titanium alloy on ageing: Influence of heating rate and chemical composition of the beta-metastable phase." Solid State Phenomena. Vol. 172. Trans Tech Publications, 2011.

[5] Lhadi, S, et al. "Micromechanical modeling of the effect of elastic and plastic anisotropies on the mechanical behavior of $\beta$-Ti alloys." International Journal of Plasticity 109 (2018): 88-107.

[6] Hounkpati, V, et al. "In situ neutron measurements and modelling of the intergranular strains in the near- $\beta$ titanium alloy Tiß21S." Acta Materialia 109 (2016): 341-352.

[7] Hémery, S., and P. Villechaise. "Influence of $\beta$ anisotropy on deformation processes operating in Ti-5Al-5Mo-5V-3Cr at room temperature." Acta Materialia 141 (2017): 285-293.

[8] Bridier, F., P. Villechaise, and J. Mendez. "Slip and fatigue crack formation processes in an $\alpha / \beta$ titanium alloy in relation to crystallographic texture on different scales." Acta Materialia 56.15 (2008): 3951-3962.

[9] Inal, K., J. L. Lebrun, and M. Belassel. "Second-order stresses and strains in heterogeneous steels: Self-consistent modeling and X-ray diffraction analysis." Metallurgical and materials transactions A 35.8 (2004): 2361-2369.

[10] Raghunathan, S. L., et al. "Micromechanics of Ti-10V-2Fe-3Al: In situ synchrotron characterisation and modelling." Acta Materialia 55.20 (2007): 6861-6872.

[11] Stapleton, Adam M., et al. "Evolution of lattice strain in Ti-6Al-4V during tensile loading at room temperature." Acta Materialia 56.20 (2008): 6186-6196.

[12] Cho, J. R., et al. "Intergranular strain accumulation in a near-alpha titanium alloy during plastic deformation." Acta materialia 50.19 (2002): 4847-4864.

[13] Gloaguen, D, et al. "Intergranular strain evolution in titanium during tensile loading: neutron diffraction and polycrystalline model." Metallurgical and Materials Transactions A 46.11 (2015): 5038-5046.

[14] Lhadi S., Richeton T., Berbenni S., Perroud O. Germain L., Gey N., Impact of the microstructure on the mechanical behavior of Ti-10-2-3 alloy: experiments and micromechanical modeling. In preparation (2019).

[15] Lhadi, S, et al. "Micromechanical Modeling of the Elasto-Viscoplastic Behavior and Incompatibility Stresses of $\beta$-Ti Alloys." Materials 11.7 (2018): 1227. 
[16] Méric, L, Philippe P, and Cailletaud G. "Single crystal modeling for structural calculations: part 1-model presentation." Journal of Engineering Materials and Technology 113.1 (1991): 162-170.

[17] Mareau, C. , Berbenni, S. "An affine formulation for the self-consistent modeling of elasto-viscoplastic heterogeneous materials based on the translated field method." International Journal of Plasticity 64 (2015): 134-150.

[18] Hearmon, R. F. S. "The elastic constants of crystals and other anisotropic materials." Landolt-Bornstein Tables, III/18 (1984): 1154.

[19] Martin, G., Naze, L., Cailletaud, G. "Numerical multi-scale simulations of the mechanical behavior of $\beta$-metastable titanium alloys Ti5553 and Ti17." Procedia Engineering 10 (2011): 1803-1808.

[20] Duval, Thimothée. Analyse multi-échelles des relations microstructure/propriétés mécaniques sous sollicitation monotone et cyclique des alliages de titane $\beta$-métastable. Diss. ISAE-ENSMA Ecole Nationale Supérieure de Mécanique et d'AérotechiquePoitiers, 2013.

[21] Van Swygenhoven, H, and Van Petegem S. "In-situ mechanical testing during X-ray diffraction." Materials Characterization 78 (2013): 47-59. 